\title{
COMPLETENESS OF A FERMIONIC BASIS IN THE HOMOGENEOUS XXZ MODEL
}

\author{
H. BOOS, M. JIMBO, T. MIWA AND F. SMIRNOV
}

\begin{abstract}
With the aid of the creation operators introduced in our previous works, we show how to construct a basis of the space of quasi-local operators for the homogeneous XXZ chain.
\end{abstract}

\section{INTRODUCTION}

The present note is a supplement to our previous papers [1]-3] on the fermionic structure in the XXZ model. Our aim here is to give a proof of the completeness statement announced in [3] concerning the fermionic basis of quasi-local operators.

The space of states of the XXZ model on the infinite lattice is formally the tensor product $\otimes_{j=-\infty}^{\infty} V_{j}$, where $V_{j}$ is a copy of the spin $1 / 2$ evaluation module of $U_{q}^{\prime}\left(\widehat{\mathfrak{s}}_{2}\right)$. Of interest is the expected values of local operators, i.e., those which act as identity on $V_{j}$ for all but a finite number of $j$. A quasi-local operator $X$ is one which acts on almost all $V_{j}$ as

$$
q^{2(\alpha-s) S(0)}, \quad S(k)=\frac{1}{2} \sum_{j=-\infty}^{k} \sigma_{j}^{3} .
$$

Here $q^{\alpha}$ is a parameter and $s \in \mathbb{Z}$ is the spin of $X$. In other words, $X$ is quasi-local with spin $s$ if

$$
X=q^{2(\alpha-s) S(0)} \mathcal{O}
$$

where $\mathcal{O}$ is local and $[S(\infty), \mathcal{O}]=s \mathcal{O}$. The space of all quasi-local operators (1.1) with spin $s$ is denoted by $\mathcal{W}_{s}^{(\alpha)}$.

In 1, 2], we have introduced a set of linear operators

$$
\mathbf{t}_{p}^{*}, \mathbf{b}_{p}, \mathbf{c}_{p}, \mathbf{b}_{p}^{*}, \mathbf{c}_{p}^{*} \quad(p \geq 1),
$$

which act on $\mathcal{W}^{(\alpha)}=\oplus_{s \in \mathbb{Z}} \mathcal{W}_{s}^{(\alpha)}$. Among them, $\mathbf{b}_{p}, \mathbf{c}_{p}$ annihilate the 'vacuum' $q^{2 \alpha S(0)}$, while the others can be used to create a family of elements of $\mathcal{W}^{(\alpha)}$ :

$$
\begin{aligned}
& \left(\mathbf{t}_{1}^{*}\right)^{p} \mathbf{t}_{i_{1}}^{*} \cdots \mathbf{t}_{i_{r}}^{*} \mathbf{b}_{j_{1}}^{*} \cdots \mathbf{b}_{j_{s}}^{*} \mathbf{c}_{k_{1}}^{*} \cdots \mathbf{c}_{k_{t}}^{*}\left(q^{2 \alpha S(0)}\right) \\
& \left(i_{1} \geq \cdots \geq i_{r} \geq 2, j_{1}>\cdots>j_{s} \geq 1, k_{1}>\cdots>k_{t} \geq 1, p \in \mathbb{Z}, r, s, t \geq 0\right) .
\end{aligned}
$$

The main result of [2] states that, at zero-temperature, the normalized expected values of the generating functions of (1.3) can be described explicitly in terms of

Date: October 29, 2018. 
determinants. This result has been generalized in [3] to the case of a non-zero temperature. In principle, one has therefore a complete knowledge about the expected value of an arbitrary quasi-local operator, provided that (1.3) gives a basis of $\mathcal{W}^{(\alpha)}$.

Using the (anti-)commutation relations among (1.2), it is fairly straightforward to show that the elements (1.3) are linearly independent (see Corollary 2.2 below). Hence the issue is that of the completeness, i.e., whether (1.3) span the whole space $\mathcal{W}^{(\alpha)}$. We show here that this is indeed the case. Our proof rests on the commutation relations whose proof is partly unfinished, so the completeness is settled modulo this point.

The plan of the text is as follows. In Section 2, we give a summary of known facts about the operators (1.2). We also introduce an alternative set of creation operators $\overline{\mathbf{b}}_{p}^{*}, \overline{\mathbf{c}}_{p}^{*}$. While $\mathbf{b}_{p}^{*}, \mathbf{c}_{p}^{*}$ enlarge the support of the operand to the right, $\overline{\mathbf{b}}_{p}^{*}, \overline{\mathbf{c}}_{p}^{*}$ do the same but to the left. Using these operators, we construct in Section 3 a set of elements supported on a given interval, and show that they span the space of quasi-local operators.

Throughout the text, $q$ is a non-zero complex number. Except in Lemma 2.1 we assume that $q$ is not a root of unity. We work over the base field $\mathbb{C}\left(q^{\alpha}\right), q^{\alpha}$ being an indeterminate.

\section{Preliminaries}

In this section we recall the main features of the creation and annihilation operators (1.2). Their construction is rather involved and occupies a large part of [2]. Leaving the details to that paper, we give below a summary of their properties.

2.1. Commutation relations. In the algebra generated by (1.2), $\mathbf{t}_{p}^{*}$ are central:

$$
\left[\mathbf{t}_{p}^{*}, \mathbf{x}_{p^{\prime}}\right]=0 \quad\left(p, p^{\prime} \geq 1, \mathbf{x}=\mathbf{t}^{*}, \mathbf{b}, \mathbf{c}, \mathbf{b}^{*}, \mathbf{c}^{*}\right) .
$$

The rest of the operators obey the canonical anti-commutation relations

$$
\begin{aligned}
& {\left[\mathbf{b}_{p}, \mathbf{b}_{p^{\prime}}\right]_{+}=\left[\mathbf{b}_{p}, \mathbf{c}_{p^{\prime}}\right]_{+}=\left[\mathbf{c}_{p}, \mathbf{c}_{p^{\prime}}\right]_{+}=0,} \\
& {\left[\mathbf{b}_{p}^{*}, \mathbf{b}_{p^{\prime}}\right]_{+}=\left[\mathbf{c}_{p}^{*}, \mathbf{c}_{p^{\prime}}\right]_{+}=\delta_{p, p^{\prime}}, \quad\left[\mathbf{b}_{p}^{*}, \mathbf{c}_{p^{\prime}}\right]_{+}=\left[\mathbf{c}_{p}^{*}, \mathbf{b}_{p^{\prime}}\right]_{+}=0,} \\
& {\left[\mathbf{b}_{p}^{*}, \mathbf{b}_{p^{\prime}}^{*}\right]_{+}=\left[\mathbf{b}_{p}^{*}, \mathbf{c}_{p^{\prime}}^{*}\right]_{+}=\left[\mathbf{c}_{p}^{*}, \mathbf{c}_{p^{\prime}}^{*}\right]_{+}=0 .}
\end{aligned}
$$

The relations (2.1) $-(2.3)$ are proved in [2]. At this writing, proof of the last set of relations (2.4) remains unavailable. In what follows we shall assume (2.4).

2.2. Support property. For an element $X \in \mathcal{W}_{s}^{(\alpha)}$, its support $\operatorname{supp} X$ is the minimal interval $[k, m]$ outside which $X$ coincides with $q^{2(\alpha-s) S(k-1)}$. We denote by $\left(\mathcal{W}^{(\alpha)}\right)_{[k, m]}$ the subspace of $\mathcal{W}^{(\alpha)}$ consisting of elements $X$ such that supp $X \subset[k, m]$. Sometimes we abuse the language and say that a local operator $\mathcal{O}$ is supported on $[k, m]$ if it acts as identity outside $[k, m]$. The operators (1.2) respect the support of the operand in the following sense. 
The operators $\mathbf{b}_{p}, \mathbf{c}_{p}$ preserve the support. More specifically, we have for any $X \in\left(\mathcal{W}^{(\alpha)}\right)_{[k, m]}$

$$
\begin{aligned}
& \operatorname{supp} \mathbf{x}_{p}(X) \subset[k, m] \quad(\mathbf{x}=\mathbf{b}, \mathbf{c}) \\
& \mathbf{x}_{p}(X)=0 \quad \text { if } p>m-k+1 \quad(\mathbf{x}=\mathbf{b}, \mathbf{c}) .
\end{aligned}
$$

The second property justifies to call them annihilation operators.

In contrast, the creation operators $\mathbf{t}_{p}^{*}, \mathbf{b}_{p}^{*}, \mathbf{c}_{p}^{*}$ enlarge the support according to the rule

$$
\operatorname{supp} \mathbf{x}_{p}^{*}(X) \subset[k, m+p] \quad\left(\mathbf{x}^{*}=\mathbf{t}^{*}, \mathbf{b}^{*}, \mathbf{c}^{*}\right) .
$$

Notice that the support is enlarged only to the right.

2.3. Independence of monomials. Among the operators $\mathbf{t}_{p}^{*}, \boldsymbol{\tau}=\mathbf{t}_{1}^{*} / 2$ plays a special role: it is the translation to the right by one lattice unit. In particular (2.1) tells that all operators are translationally invariant.

Separating $\mathbf{t}_{1}^{*}$ from the rest, we set

$$
\mathbf{h}_{p}^{*}=\left(\mathbf{t}_{1}^{*}\right)^{-1} \mathbf{t}_{p+1}^{*}, \quad \mathbf{h}^{*}(\zeta)=\sum_{p=0}^{\infty}\left(\zeta^{2}-1\right)^{p} \mathbf{h}_{p}^{*} .
$$

Accodring to [2], Subsection $3.4, \mathbf{h}^{*}(\zeta)$ is given by the following formula. For $X \in$ $\left(\mathcal{W}^{(\alpha)}\right)_{[k, m]}$, we have

$$
\mathbf{h}^{*}(\zeta)(X)=\lim _{l \rightarrow \infty} \widetilde{\mathbb{R}}_{l, l-1}^{\vee}\left(\zeta^{2}\right) \cdots \cdots \widetilde{\mathbb{R}}_{k, k-1}^{\vee}\left(\zeta^{2}\right)(X) .
$$

Here $\widetilde{\mathbb{R}}_{i+1, i}^{\vee}\left(\zeta^{2}\right) \in \operatorname{End}\left(V_{i} \otimes V_{i+1}\right)$ denotes the adjoint action by the standard $R$ matrix for the XXZ model. We have $\widetilde{\mathbb{R}}_{i+1, i}^{\vee}(1)=1$. Moreover if $\operatorname{supp}(X) \subset[a, b]$ with either $i>b$ or $i<a-1$, then $\widetilde{\mathbb{R}}_{i+1, i}^{\vee}\left(\zeta^{2}\right)(X)=X$ holds. These properties of $\widetilde{\mathbb{R}}_{i+1, i}^{\vee}\left(\zeta^{2}\right)$ ensure that each coefficient of the series appearing in the right hand side of (2.8) stabilizes for large enough $l$. More precisely, we have for any $p$

$$
\mathbf{h}^{*}(\zeta)(X) \equiv \widetilde{\mathbb{R}}_{m+p, m+p-1}^{\vee}\left(\zeta^{2}\right) \cdots \cdots \widetilde{\mathbb{R}}_{k, k-1}^{\vee}\left(\zeta^{2}\right)(X) \quad \bmod \left(\zeta^{2}-1\right)^{p+1} .
$$

Consequently we have the support property

$$
\operatorname{supp} \mathbf{h}_{p}^{*}(X) \subset[k-1, m+p] .
$$

Lemma 2.1. If $q$ is generic, then the set of elements

$$
\left(\mathbf{h}_{1}^{*}\right)^{m_{1}}\left(\mathbf{h}_{2}^{*}\right)^{m_{2}} \cdots\left(q^{2 \alpha S(0)}\right) \quad\left(m_{1}, m_{2}, \cdots \geq 0\right)
$$

is linearly independent.

Proof. It is enough to show the assertion for a special value of the parameter $q$. We choose $q=\sqrt{-1}$, writing $q^{\alpha}$ as $y$ and $q^{2 \alpha S(0)}$ as $y^{2 S(0)}$.

Following [1], let us introduce linear operators

$$
\Psi_{j}^{ \pm}, \Phi_{j}^{ \pm}: \mathcal{W}_{s}^{(\alpha)} \longrightarrow \mathcal{W}_{s \pm 1}^{(\alpha)}
$$


by the formula

$$
\begin{aligned}
& \Psi_{j}^{ \pm}(X)=\psi_{j}^{ \pm} X-(-1)^{s} X \psi_{j}^{ \pm}, \\
& \Phi_{j}^{ \pm}(X)=\frac{1}{1-y^{\mp 2}}\left(\psi_{j}^{ \pm} X-y^{\mp 2}(-1)^{s} X \psi_{j}^{ \pm}\right),
\end{aligned}
$$

where $X \in \mathcal{W}_{s}^{(\alpha)}$ and

$$
\psi_{j}^{ \pm}=\sigma_{j}^{ \pm} e^{\mp \pi i S(j-1)} .
$$

They satisfy the canonical anti-commutation relations

$$
\left[\Psi_{j}^{\epsilon}, \Psi_{k}^{\epsilon^{\prime}}\right]_{+}=0, \quad\left[\Phi_{j}^{\epsilon}, \Phi_{k}^{\epsilon^{\prime}}\right]_{+}=0, \quad\left[\Psi_{j}^{\epsilon}, \Phi_{k}^{\epsilon^{\prime}}\right]_{+}=\delta_{j, k} \delta_{0, \epsilon+\epsilon^{\prime}}
$$

as well as the annihilation property

$$
\begin{array}{ll}
\Psi_{j}^{ \pm}\left(y^{2 S(0)}\right)=0 & (j>0), \\
\Phi_{j}^{ \pm}\left(y^{2 S(0)}\right)=0 & (j \leq 0) .
\end{array}
$$

Acting with the fermions $\Psi_{j}^{ \pm}, \Phi_{j}^{ \pm}$over the vacuum $y^{2 S(0)}$ we obtain a Fock space $\mathcal{F} \subset \mathcal{W}^{(\alpha)}$.

When $q=\sqrt{-1}$, the $R$ matrix simplifies to

$$
\begin{aligned}
& \widetilde{\mathbb{R}}_{i+1, i}^{\vee}\left(\zeta^{2}\right)(X)=e^{H_{i, i+1}} X e^{-H_{i, i+1}}, \\
& H_{i, i+1}=z\left(\psi_{i}^{+}+\psi_{i+1}^{+}\right)\left(\psi_{i}^{-}-\psi_{i+1}^{-}\right),
\end{aligned}
$$

where

$$
z=\frac{1-\zeta^{2}}{1+\zeta^{2}}
$$

Expressing the left and right multiplication operators by $\psi_{j}^{ \pm}$in terms of $\Psi_{j}^{ \pm}$and $\Phi_{j}^{ \pm}$, we obtain an identity of linear operators on $\mathcal{F}$

$$
\widetilde{\mathbb{R}}_{i+1, i}^{\vee}\left(\zeta^{2}\right)=\exp \left(z \sum_{\epsilon= \pm}\left(\epsilon \Phi_{i}^{\epsilon}+\Phi_{i+1}^{\epsilon}\right)\left(\Psi_{i}^{-\epsilon}-\epsilon \Psi_{i+1}^{-\epsilon}\right)\right) .
$$

A simple calculation using the definition (2.8) shows that as formal power series in $z$ we have

$$
\begin{aligned}
& \mathbf{h}^{*}(\zeta) \Phi_{p}^{ \pm} \mathbf{h}^{*}(\zeta)^{-1}=-\Phi_{p-1}^{ \pm} z+\left(1-z^{2}\right) \sum_{k \geq p} \Phi_{k}^{ \pm} z^{k-p} \\
& \mathbf{h}^{*}(\zeta) \Psi_{p}^{ \pm} \mathbf{h}^{*}(\zeta)^{-1}=-\Psi_{p-1}^{ \pm} z+\left(1-z^{2}\right) \sum_{k \geq p} \Psi_{k}^{ \pm} z^{k-p}
\end{aligned}
$$

The Fock vacuum expectation value of $\mathbf{h}^{*}(\zeta)$ is easily evaluated, with the result

$$
\left\langle\mathbf{h}^{*}(\zeta)\right\rangle=1-z^{2} \text {. }
$$

The operator $\mathbf{h}^{*}(\zeta)$ is characterized by (2.13), (2.14) and (2.15). We thus find the expression on $\mathcal{F}$

$$
\mathbf{h}^{*}(\zeta)=\left(1-z^{2}\right) \exp \left(\sum_{\nu=1}^{\infty} \frac{z^{\nu}}{\nu}\left(\mathcal{J}_{-\nu}-\mathcal{J}_{\nu}\right)\right)
$$


where we used the standard bosonization formula

$$
\mathcal{J}_{\nu}=\sum_{\epsilon= \pm} \sum_{p \in \mathbb{Z}}: \Phi_{p}^{\epsilon} \Psi_{p+\nu}^{-\epsilon}:
$$

which satisfies

$$
\begin{aligned}
& {\left[\mathcal{J}_{\mu}, \mathcal{J}_{\nu}\right]=2 \mu \delta_{\mu+\nu, 0},} \\
& \mathcal{J}_{\nu}\left(y^{2 S(0)}\right)=0 \quad(\nu>0) .
\end{aligned}
$$

Since the coefficients of the expansion of $\log \mathbf{h}^{*}(\zeta)$ belong to the creation part of the Heisenberg algebra, the statement of Proposition is obvious.

Corollary 2.2. The elements (1.3) are linearly independent.

Proof. This is a direct consequence of Lemma 2.1 and the commutation relations (2.1) and (2.3).

2.4. Another family of operators. In addition to $\mathbf{b}_{p}, \mathbf{c}_{p}$, we have considered in [2] another set of annihilation operators $\overline{\mathbf{b}}_{p}, \overline{\mathbf{c}}_{p}$. They have the same support property

$$
\begin{aligned}
& \operatorname{supp} \overline{\mathbf{x}}_{p}(X) \subset[k, m] \quad(\overline{\mathbf{x}}=\overline{\mathbf{b}}, \overline{\mathbf{c}}), \\
& \overline{\mathbf{x}}_{p}(X)=0 \quad \text { if } p>m-k+1 \quad(\overline{\mathbf{x}}=\overline{\mathbf{b}}, \overline{\mathbf{c}}),
\end{aligned}
$$

anti-commute with $\mathbf{b}_{p^{\prime}}, \mathbf{c}_{p^{\prime}}$ and satisfy

$$
\left[\mathbf{b}_{p}^{*}, \overline{\mathbf{b}}_{p^{\prime}}\right]_{+}=\left[\mathbf{c}_{p}^{*}, \overline{\mathbf{c}}_{p^{\prime}}\right]_{+}=-\mathbf{t}_{p-p^{\prime}+1}^{*} .
$$

In fact, they can be expressed in terms of the operators (1.2) as follows (see [3], Corollary A.2):

$$
\left(\mathbf{t}_{1}^{*}\right)^{-1} \overline{\mathbf{b}}_{p}=-\sum_{p^{\prime} \geq 0} \mathbf{h}_{p^{\prime}}^{*} \mathbf{b}_{p+p^{\prime}}, \quad\left(\mathbf{t}_{1}^{*}\right)^{-1} \overline{\mathbf{c}}_{p}=-\sum_{p^{\prime} \geq 0} \mathbf{h}_{p^{\prime}}^{*} \mathbf{c}_{p+p^{\prime}}
$$

Due to (2.6), the sum in the right hand side is finite on each operand $X \in \mathcal{W}^{(\alpha)}$. Even though these operators are not independent, we find it useful to take them into consideration.

Let us introduce the creation counterpart to these operators. Define $\overline{\mathbf{b}}_{p}^{*}, \overline{\mathbf{c}}_{p}^{*}(p \geq 1)$ inductively by

$$
\mathbf{b}_{p}^{*}=\sum_{p^{\prime}=1}^{p} \mathbf{h}_{p-p^{\prime}}^{*} \overline{\mathbf{b}}_{p^{\prime}}^{*}, \quad \mathbf{c}_{p}^{*}=\sum_{p^{\prime}=1}^{p} \mathbf{h}_{p-p^{\prime}}^{*} \overline{\mathbf{c}}_{p^{\prime}}^{*}
$$

We have then

$$
\left[\overline{\mathbf{b}}_{p}^{*},\left(\mathbf{t}_{1}^{*}\right)^{-1} \overline{\mathbf{b}}_{p^{\prime}}\right]_{+}=\left[\overline{\mathbf{c}}_{p}^{*},\left(\mathbf{t}_{1}^{*}\right)^{-1} \overline{\mathbf{c}}_{p^{\prime}}\right]_{+}=-\delta_{p, p^{\prime}} .
$$

In the sequel we consider the generating series

$$
\begin{aligned}
& \mathbf{x}(\zeta)=\sum_{p \geq 1}\left(\zeta^{2}-1\right)^{-p} \mathbf{x}_{p} \quad(\mathbf{x}=\mathbf{b}, \mathbf{c}, \overline{\mathbf{b}}, \overline{\mathbf{c}}) \\
& \mathbf{x}^{*}(\zeta)=\sum_{p \geq 1}\left(\zeta^{2}-1\right)^{p-1} \mathbf{x}_{p}^{*} \quad\left(\mathbf{x}^{*}=\mathbf{b}^{*}, \mathbf{c}^{*}, \overline{\mathbf{b}}^{*}, \overline{\mathbf{c}}^{*}\right) .
\end{aligned}
$$


Then we have the relations of formal series

$$
\begin{aligned}
& \overline{\mathbf{x}}(\zeta) \equiv-\mathbf{t}_{1}^{*} \mathbf{h}^{*}(\zeta) \mathbf{x}(\zeta) \quad \bmod \left(\zeta^{2}-1\right)^{0}, \\
& \overline{\mathbf{x}}^{*}(\zeta)=\mathbf{h}^{*}(\zeta)^{-1} \mathbf{x}^{*}(\zeta)
\end{aligned}
$$

Unlike $\mathbf{b}_{p}^{*}, \mathbf{c}_{p}^{*}$, they enlarge the support essentially to the left.

Proposition 2.3. For any $X \in\left(\mathcal{W}^{(\alpha)}\right)_{[k, m]}$ we have

$$
\operatorname{supp} \overline{\mathbf{x}}_{p}^{*}(X) \subset[k-p+1, m+1] \quad\left(\overline{\mathbf{x}}^{*}=\overline{\mathbf{b}}^{*}, \overline{\mathbf{c}}^{*}\right) .
$$

Proof. It is known (see [2], Lemma 3.7) that $\mathbf{b}^{*}(\zeta)(X)$ has an expression

$$
\mathbf{b}^{*}(\zeta)(X)=\lim _{l \rightarrow \infty} \widetilde{\mathbb{R}}_{l, l-1}^{\vee}\left(\zeta^{2}\right) \cdots \cdots \widetilde{\mathbb{R}}_{m+2, m+1}^{\vee}\left(\zeta^{2}\right)(Y(\zeta))
$$

for some $Y(\zeta) \in\left(\mathcal{W}^{(\alpha)}\right)_{[k, m+1]}$. Using the formula

$$
\mathbf{h}^{*}(\zeta)^{-1}\left(X^{\prime}\right)=\lim _{l \rightarrow \infty} \widetilde{\mathbb{R}}_{-l+1,-l}^{\vee}\left(\zeta^{2}\right)^{-1} \cdots \cdots \widetilde{\mathbb{R}}_{m^{\prime}+1, m^{\prime}}^{\vee}\left(\zeta^{2}\right)^{-1}\left(X^{\prime}\right)
$$

valid for any $X^{\prime} \in\left(\mathcal{W}^{(\alpha)}\right)_{\left[k^{\prime}, m^{\prime}\right]}$, we obtain that

$$
\overline{\mathbf{b}}^{*}(\zeta)(X)=\lim _{l \rightarrow \infty} \widetilde{\mathbb{R}}_{-l+1,-l}^{\vee}\left(\zeta^{2}\right)^{-1} \cdots \cdots \widetilde{\mathbb{R}}_{k, k-1}^{\vee}\left(\zeta^{2}\right)^{-1} \cdots \cdots \widetilde{\mathbb{R}}_{m+1, m}^{\vee}\left(\zeta^{2}\right)^{-1}(Y(\zeta)) .
$$

By the same argument as for (2.9), this expression implies that the support property $\overline{\mathbf{b}}_{p}^{*}(X) \subset[k-p+1, m+1]$ holds.

The case of $\overline{\mathbf{c}}_{p}^{*}$ is entirely similar.

Remark. To simplify the notation, in this paper we have modified the definition of the generating functions given in [2]. Denoting those in [2] by $\mathbf{b}_{I I}^{*}(\zeta)$, etc., the present definition is related to them as follows.

$$
\begin{aligned}
& \mathbf{b}_{I I}(\zeta)=\zeta^{-\alpha-\mathbb{S}}\left(\mathbf{b}_{0}+\mathbf{b}(\zeta)\right), \\
& \mathbf{c}_{I I}(\zeta)=\zeta^{\alpha+\mathbb{S}}\left(\mathbf{c}_{0}+\mathbf{c}(\zeta)\right), \\
& \mathbf{b}_{I I}^{*}(\zeta)=\zeta^{\alpha+\mathbb{S}+2} \mathbf{b}^{*}(\zeta), \\
& \mathbf{c}_{I I}^{*}(\zeta)=\zeta^{-\alpha-\mathbb{S}-2} \mathbf{c}^{*}(\zeta) .
\end{aligned}
$$

\section{A BASIS OF $\left(\mathcal{W}^{(\alpha)}\right)_{[1, n]}$}

The goal of this section is to construct a basis of $\left(\mathcal{W}^{(\alpha)}\right)_{[k, m]}$ for all intervals $[k, m]$ using linear combinations of (1.3). In view of the translational invariance we concentrate on the case $[1, n]$.

In order to verify the spanning property, one has to find sufficiently many operators supported on $[1, n]$. In general the monomials (1.3) have too large support, and suitable linear combinations of them must be chosen. We do this in two steps. First we introduce certain elements $B_{J}$ and show that their support is contained in $[1, n]$. We then construct general basis elements by applying to them annihilation operators which do not enlarge the support. 
3.1. Elements $B_{J}$. Let $l$ be a non-negative integer satisfying $n \geq l \geq 0$. We define the numbers

$$
C_{j_{1}, \cdots, j_{l} ; k_{1}, \cdots, k_{l}}^{i_{1}, \cdots, i_{l}} \quad\left(1 \leq i_{p}, j_{p}, k_{p}\right)
$$

by the generating series

$$
\frac{\Delta(x) \Delta(y) \Delta(z)}{\prod_{i, j=1}^{l}\left(1-x_{i} y_{j}\right)\left(1-x_{i} z_{j}\right)}=\sum C_{j_{1}, \cdots, j_{l}, k_{1}, \cdots, k_{l}}^{i_{1}, \cdots, i_{l}} \prod_{p=1}^{l}\left(x_{p}^{i_{p}-1} y_{p}^{j_{p}-1} z_{p}^{k_{p}-1}\right) .
$$

Here $x=\left(x_{1}, \cdots, x_{l}\right), y=\left(y_{1}, \cdots, y_{l}\right), z=\left(z_{1}, \cdots, z_{l}\right), \Delta(x)=\prod_{1 \leq i<j \leq l}\left(x_{i}-x_{j}\right)$, and the sum is taken over all positive integers $i_{p}, j_{p}, k_{p}(p=1, \cdots, l)$.

When all sequences are decreasing $\left(i_{1}>\cdots>i_{l}\right.$, etc. $)$, we identify them with subsets $I=\left\{i_{1}, \cdots, i_{l}\right\} \subset[1, n]$, etc., and write $C_{J, K}^{I}$ for $(3.1)$. We shall write $|I|$ for the cardinality of $I$. In this case $C_{J, K}^{I}$ coincides with the Littlewood-Richardson coefficient $c_{\mu, \nu}^{\lambda}$ well-known in combinatorics (see [5], eq.(5.2)); in particular they are non-negative integers. The precise correspondence reads

$$
C_{J, K}^{I}=c_{\lambda(J), \lambda(K)}^{\lambda(I)},
$$

where $\lambda(I)=\left(\lambda_{1}, \cdots, \lambda_{l}\right)=\left(i_{1}-l, \cdots, i_{l}-1\right)$.

In what follows, for a subset $I=\left\{i_{1}, \cdots, i_{l}\right\}\left(i_{1}>\cdots>i_{l}\right)$ of $[1, n]$, we write

$$
\mathbf{x}_{M}=\mathbf{x}_{i_{1}} \cdots \mathbf{x}_{i_{l}}
$$

for $\mathbf{x}=\mathbf{b}, \cdots, \overline{\mathbf{c}}^{*}$.

Now we introduce a family of operators $B_{J}$ indexed by $J \subset[1, n]$. Writing $l=|J|$ we define

$$
B_{J}=\sum_{I, K} C_{J, K}^{I} \mathbf{b}_{n}^{*} \cdots \mathbf{c}_{k_{1}}^{i_{1}} \cdots \mathbf{c}_{k_{l}}^{i_{l}} \cdots \mathbf{b}_{1}^{*}\left(q^{2 \alpha S(0)}\right) .
$$

In the last line, the sum is taken over all subsets $I, K \subset[1, n]$ with $I=\left\{i_{1}, \cdots, i_{l}\right\}$, $K=\left\{k_{1}, \cdots, k_{l}\right\}$, and $\mathbf{c}_{k_{p}}^{*}$ is placed at the $i_{p}$-th slot.

Since $c_{\mu, \emptyset}^{\lambda}=\delta_{\lambda, \mu}$, we have

$$
B_{J}= \pm \mathbf{b}_{\complement J}^{*} \mathbf{c}_{\{1, \cdots, l\}}^{*}\left(q^{2 \alpha S(0)}\right)+\cdots
$$

where $\cdots$ stands for terms which do not contain $\mathbf{c}_{\{1, \cdots, l\}}^{*}$.

Example. Let $n=4$ and $l=2$. Suppressing $q^{2 \alpha S(0)}$ we have

$$
\begin{aligned}
B_{\{3,4\}} & =\mathbf{c}_{2}^{*} \mathbf{c}_{1}^{*} \mathbf{b}_{2}^{*} \mathbf{b}_{1}^{*}, \\
B_{\{2,4\}} & =\mathbf{c}_{2}^{*} \mathbf{b}_{3}^{*} \mathbf{c}_{1}^{*} \mathbf{b}_{1}^{*}+\mathbf{c}_{3}^{*} \mathbf{c}_{1}^{*} \mathbf{b}_{2}^{*} \mathbf{b}_{1}^{*}, \\
B_{\{1,4\}} & =\mathbf{c}_{2}^{*} \mathbf{b}_{3}^{*} \mathbf{b}_{2}^{*} \mathbf{c}_{1}^{*}+\mathbf{c}_{3}^{*} \mathbf{b}_{3}^{*} \mathbf{c}_{1}^{*} \mathbf{b}_{1}^{*}+\mathbf{c}_{4}^{*} \mathbf{c}_{1}^{*} \mathbf{b}_{2}^{*} \mathbf{b}_{1}^{*}, \\
B_{\{2,3\}} & =\mathbf{b}_{4}^{*} \mathbf{c}_{2}^{*} \mathbf{c}_{1}^{*} \mathbf{b}_{1}^{*}+\mathbf{c}_{3}^{*} \mathbf{b}_{3}^{*} \mathbf{c}_{1}^{*} \mathbf{b}_{1}^{*}+\mathbf{c}_{3}^{*} \mathbf{c}_{2}^{*} \mathbf{b}_{2}^{*} \mathbf{b}_{1}^{*}, \\
B_{\{1,3\}} & =\mathbf{b}_{4}^{*} \mathbf{c}_{2}^{*} \mathbf{b}_{2}^{*} \mathbf{c}_{1}^{*}+\mathbf{c}_{3}^{*} \mathbf{b}_{3}^{*} \mathbf{b}_{2}^{*} \mathbf{c}_{1}^{*}+\mathbf{b}_{4}^{*} \mathbf{c}_{3}^{*} \mathbf{c}_{1}^{*} \mathbf{b}_{1}^{*}+\mathbf{c}_{3}^{*} \mathbf{b}_{3}^{*} \mathbf{c}_{2}^{*} \mathbf{b}_{1}^{*}+\mathbf{c}_{4}^{*} \mathbf{b}_{3}^{*} \mathbf{c}_{1}^{*} \mathbf{b}_{1}^{*}+\mathbf{c}_{4}^{*} \mathbf{c}_{2}^{*} \mathbf{b}_{2}^{*} \mathbf{b}_{1}^{*}, \\
B_{\{1,2\}} & =\mathbf{b}_{4}^{*} \mathbf{b}_{3}^{*} \mathbf{c}_{2}^{*} \mathbf{c}_{1}^{*}+\mathbf{b}_{4}^{*} \mathbf{c}_{3}^{*} \mathbf{b}_{2}^{*} \mathbf{c}_{1}^{*}+\mathbf{c}_{4}^{*} \mathbf{b}_{3}^{*} \mathbf{b}_{2}^{*} \mathbf{c}_{1}^{*}+\mathbf{b}_{4}^{*} \mathbf{c}_{3}^{*} \mathbf{c}_{2}^{*} \mathbf{b}_{1}^{*}+\mathbf{c}_{4}^{*} \mathbf{b}_{3}^{*} \mathbf{c}_{2}^{*} \mathbf{b}_{1}^{*}+\mathbf{c}_{4}^{*} \mathbf{c}_{3}^{*} \mathbf{b}_{2}^{*} \mathbf{b}_{1}^{*} .
\end{aligned}
$$


These elements admit an alternative expression in terms of the other set of creation operators.

Lemma 3.1. We have

$$
B_{J}=\sum_{I, K} C_{J, K}^{I} \overline{\mathbf{b}}_{n}^{*} \cdots \overline{\mathbf{c}}_{k_{1}}^{i_{1}} \cdots \overline{\mathbf{c}}_{k_{l}}^{i_{l}} \cdots \overline{\mathbf{b}}_{1}^{*}\left(q^{2 \alpha S(0)}\right) .
$$

Proof. First we note that the definition (3.3) can be written as

$$
\begin{aligned}
B_{J} & =\frac{1}{l !^{2}}\left(\sum_{\substack{n \geq i_{p}, k_{p} \geq 1 \\
1 \leq p \leq l}} C_{J, K}^{I} \mathbf{c}_{k_{1}}^{*} \mathbf{b}_{i_{1}} \cdots \mathbf{c}_{k_{l}}^{*} \mathbf{b}_{i_{l}}\right) B_{\emptyset}, \\
B_{\emptyset} & =\mathbf{b}_{n}^{*} \cdots \mathbf{b}_{1}^{*}\left(q^{2 \alpha S(0)}\right) .
\end{aligned}
$$

Actually the restriction $n \geq i_{p}, k_{p}$ is irrelevant, since otherwise the corresponding term is zero. Extending the suffix of $B_{J}$ by anti-symmetry, we consider their generating series. Inserting

$$
\mathbf{c}_{k}^{*} \mathbf{b}_{i}=\oint \frac{d \zeta^{2}}{2 \pi i} \frac{d \xi^{2}}{2 \pi i} \mathbf{c}^{*}(\zeta) \mathbf{b}(\xi)\left(\zeta^{2}-1\right)^{-k}\left(\xi^{2}-1\right)^{i-1}
$$

and using (3.2), we get

$$
\begin{gathered}
l !^{2} \sum_{j_{1}, \cdots, j_{l} \geq 1} B_{j_{1}, \cdots, j_{l}} \prod_{p=1}^{l} y_{p}^{j_{p}-1}=\oint \prod_{p=1}^{l} \frac{d \zeta_{p}^{2}}{2 \pi i} \frac{d \xi_{p}^{2}}{2 \pi i} \prod_{p=1}^{l} z_{p} \frac{\Delta(x) \Delta(y) \Delta(z)}{\prod_{i, j=1}^{l}\left(1-x_{i} y_{j}\right)\left(1-x_{i} z_{j}\right)} \\
\times \mathbf{c}^{*}\left(\zeta_{1}\right) \mathbf{b}\left(\xi_{1}\right) \cdots \mathbf{c}^{*}\left(\zeta_{l}\right) \mathbf{b}\left(\xi_{l}\right) B_{\emptyset} .
\end{gathered}
$$

Here we have set $z_{p}=\left(\zeta_{p}^{2}-1\right)^{-1}, x_{p}=\xi_{p}^{2}-1$, and the integral is taken along the contour encircling $\zeta_{p}^{2}=\xi_{p}^{2}=1,\left|\zeta_{p}^{2}-1\right|>\left|\xi_{p^{\prime}}^{2}-1\right|$. Noting that

$$
\frac{\Delta(x) \Delta(z)}{\prod_{i, j=1}^{l}\left(1-x_{i} z_{j}\right)}=\operatorname{det}\left(\frac{1}{\zeta_{p}^{2}-\xi_{p^{\prime}}^{2}}\right)_{1 \leq p, p^{\prime} \leq l}
$$

and integrating over $\xi_{p}^{2}$, we find

$$
\begin{aligned}
& (-1)^{l} \frac{l !}{\Delta(y)} \sum_{j_{1}, \cdots, j_{l} \geq 1} B_{j_{1}, \cdots, j_{l}} \prod_{p=1}^{l} y_{p}^{j_{p}-1} \\
& =\oint \prod_{p=1}^{l} \frac{d \zeta_{p}^{2}}{2 \pi i} \prod_{i, j=1}^{l} \frac{1}{1-y_{i}\left(\zeta_{j}^{2}-1\right)} \mathbf{c}^{*}\left(\zeta_{1}\right) \mathbf{b}\left(\zeta_{1}\right) \cdots \mathbf{c}^{*}\left(\zeta_{l}\right) \mathbf{b}\left(\zeta_{l}\right) B_{\emptyset} .
\end{aligned}
$$

On the other hand, we have

$$
\begin{aligned}
& \mathbf{c}^{*}(\zeta) \mathbf{b}(\zeta) \equiv-\overline{\mathbf{c}}^{*}(\zeta)\left(\mathbf{t}_{1}^{*}\right)^{-1} \overline{\mathbf{b}}(\zeta) \quad \bmod \left(\zeta^{2}-1\right)^{0} \\
& \mathbf{b}_{n}^{*} \cdots \mathbf{b}_{1}^{*}=\overline{\mathbf{b}}_{n}^{*} \cdots \overline{\mathbf{b}}_{1}^{*} .
\end{aligned}
$$

Noting further that $\left[\overline{\mathbf{b}}_{p}^{*},\left(\mathbf{t}_{1}^{*}\right)^{-1} \overline{\mathbf{b}}_{p^{\prime}}\right]_{+}=-\delta_{p, p^{\prime}}$, we see from (3.5) that the definition (3.3) of $B_{J}$ is unchanged if we interchange barred and unbarred operators. 
Corollary 3.2. We have

$$
\operatorname{supp} B_{J} \subset[1, n] \text {. }
$$

Proof. This follows from the two different expressions (3.3), (3.4) and the support property (2.5), (2.21):

$$
\operatorname{supp} B_{J} \subset[1, \infty) \cap(-\infty, n]=[1, n] .
$$

3.2. Construction of a basis. Consider the following set of elements of $\mathcal{W}^{(\alpha)}$ :

$$
\overline{\mathbf{b}}_{M} \mathbf{c}_{N}\left(B_{J}\right) \quad(J \subset[1, n], ; M \subset[1, n-|J|], N \subset[1,|J|]) .
$$

As $M, N, J$ vary there are altogether $4^{n}$ such elements. Due to Corollary 3.2, and since annihilation operators preserve the support, they are all supported in $[1, n]$.

Example. We omit writing $q^{2 \alpha S(0)}$. For $n=2$, we have

$$
B_{\emptyset}=\mathbf{b}_{2}^{*} \mathbf{b}_{1}^{*}, B_{\{2\}}=\mathbf{c}_{1}^{*} \mathbf{b}_{1}^{*}, B_{\{1\}}=\mathbf{b}_{2}^{*} \mathbf{c}_{1}^{*}+\mathbf{c}_{2}^{*} \mathbf{b}_{1}^{*}, B_{\{1,2\}}=\mathbf{c}_{2}^{*} \mathbf{c}_{1}^{*} .
$$

The elements (3.6) give

$$
\begin{aligned}
& \mathbf{b}_{2}^{*} \mathbf{b}_{1}^{*} ; \\
& \mathbf{b}_{2}^{*}, \mathbf{b}_{1}^{*}, \mathbf{t}_{1}^{*} \mathbf{b}_{2}^{*}-\mathbf{t}_{2}^{*} \mathbf{b}_{1}^{*}, \mathbf{t}_{1}^{*} \mathbf{b}_{1}^{*} ; \\
& 1, \mathbf{t}_{1}^{*}, \mathbf{t}_{2}^{*},\left(\mathbf{t}_{1}^{*}\right)^{2}, \mathbf{c}_{1}^{*} \mathbf{b}_{1}^{*}, \mathbf{b}_{2}^{*} \mathbf{c}_{1}^{*}+\mathbf{c}_{2}^{*} \mathbf{b}_{1}^{*} ; \\
& \mathbf{c}_{2}^{*}, \mathbf{c}_{1}^{*}, \mathbf{t}_{1}^{*} \mathbf{c}_{2}^{*}-\mathbf{t}_{2}^{*} \mathbf{c}_{1}^{*}, \mathbf{t}_{1}^{*} \mathbf{c}_{1}^{*} ; \\
& \mathbf{c}_{2}^{*} \mathbf{c}_{1}^{*}
\end{aligned}
$$

The following is the main result of this note.

Theorem 3.3. The elements (3.6) constitute a basis of $\left(\mathcal{W}^{(\alpha)}\right)_{[1, n]}$.

Proof. It is sufficient to show that the set (3.6) is linearly independent. Suppose there is a linear relation

$$
\sum_{M, N, J} A_{M, N, J} \overline{\mathbf{b}}_{M} \mathbf{c}_{N}\left(B_{J}\right)=0
$$

with some scalars $A_{M, N, J}$. The sum is taken over $J \subset[1, n], M \subset[1, n-|J|]$, $N \subset[1,|J|]$. The left hand side is a linear combination of monomials containing $l=|J|$ number of $\mathbf{b}^{*}$ and $\mathbf{t}^{*}$. Hence the sum is separately zero for each fixed $l$.

Fixing $M_{0} \subset[1, n-l], N_{0} \subset[1, l]$, we apply $\overline{\mathbf{b}}_{[1, \cdots, n-l] \backslash M_{0}} \mathbf{c}_{[1, l] \backslash N_{0}}$ to both sides. We obtain

$$
\begin{aligned}
0 & =\sum_{|J|=l} A_{M_{0}, N_{0}, J} \overline{\mathbf{b}}_{\{1, \cdots, n-l\}} \mathbf{c}_{\{1, \cdots, l\}} \\
& \times \sum_{I, K} C_{J, K}^{I} \mathbf{c}_{k_{l}}^{*} \mathbf{b}_{i_{l}} \cdots \mathbf{c}_{k_{1}}^{*} \mathbf{b}_{i_{1}} \mathbf{b}_{n}^{*} \cdots \mathbf{b}_{1}^{*}\left(q^{2 \alpha S(0)}\right) .
\end{aligned}
$$


In the second sum only the term with $K=\{1, \cdots, l\}$ contributes. Noting that $C_{J,\{1, \cdots, l\}}^{I}=\delta_{I, J}$, we obtain

$$
0=\sum_{J} A_{M_{0}, N_{0}, J} \operatorname{det}\left(\mathbf{h}_{j_{a}^{\prime}-b}^{*}\right)_{1 \leq a, b \leq n-l}\left(q^{2 \alpha S(0)}\right),
$$

where $\left\{j_{1}^{\prime}, \cdots, j_{n-l}^{\prime}\right\}$ denotes the complement of $J=\left\{j_{1}, \cdots, j_{n}\right\}$ in $[1, n]$. By Lemma 2.1, Schur functions in $\mathbf{h}_{p}^{*}$ 's applied to $q^{2 \alpha S(0)}$ are linearly independent. Hence we conclude that $A_{M_{0}, N_{0}, J}=0$ for all $J$. This completes the proof.

Acknowledgements. $\mathrm{HB}$ is grateful to the Volkswagen Foundation and to the 'Graduiertenkolleg' DFG project: "Representation theory and its application in mathematics and physics" for the financial support. Research of MJ is supported by the Grant-in-Aid for Scientific Research B-20340027 and B-20340011. Research of TM is supported by the Grant-in-Aid for Scientific Research B-17340038. Research of FS is supported by EC networks "ENIGMA", contract number MRTN-CT-20045652 This work was supported by World Premier International Research Center Initiative (WPI Initiative), MEXT, Japan.

\section{REFERENCES}

[1] H. Boos, M. Jimbo, T. Miwa, F. Smirnov and Y. Takeyama, Hidden Grassmann structure in the XXZ model, Commun. Math. Phys. 272 (2007), 263-281.

[2] H. Boos, M. Jimbo, T. Miwa, F. Smirnov and Y. Takeyama, Hidden Grassmann structure in the XXZ model II: Creation operators, to appear in Commun. Math. Phys. (2009)

[3] M. Jimbo, T. Miwa and F. Smirnov, Hidden Grassmann structure in the XXZ model III: Introducing Matsubara direction, arXiv:0811.0439, to appear in J. Phys. A:Math. Theor.

[4] H. Boos, M. Jimbo, T. Miwa, F. Smirnov and Y. Takeyama, Fermionic basis for space of operators in the XXZ model, SISSA Proceedings of Science (2007), Paper 015, 34 pp. (electronic)

[5] I. Macdonald, Symmetric functions and orthogonal polynomials, 2nd ed., Oxford University Press, 1995.

HB: Physics Department, University of Wuppertal, D-42097, Wuppertal, GerMANY

E-mail address: boos@physik.uni-wuppertal.de

MJ: Graduate School of Mathematical Sciences, The University of Tokyo, Tokyo 153-8914, JAPAN

E-mail address: jimbomic@ms.u-tokyo.ac.jp

TM: Department of Mathematics, Graduate School of Science, Kyoto UniverSITY, KYOTO 606-8502, JAPAN

E-mail address: tetsuji@math.kyoto-u.ac.jp 
FS1: Laboratoire de Physique Théorique et Hautes Energies, Université Pierre et Marie Curie, Tour $161^{\text {er }}$ Étage, 4 Place Jussieu 75252 Paris Cedex 05, France E-mail address: smirnov@lpthe.jussieu.fr

\footnotetext{
${ }^{1}$ Membre du CNRS
} 